\title{
Mixed-Reality and Traditional Flight Simulators for Urban Air Mobility Vehicles
}

\author{
Keegan Bergman ${ }^{1}$, Ryan Bowers ${ }^{2}$, Van Duong ${ }^{2}$, Torie Miller ${ }^{3}$, Austin Peek ${ }^{3}$, Mirin Morris- \\ Ward $^{4}$, Kyle Tam ${ }^{4}$, Noah Fitzpatrick ${ }^{5}$, William Grismore ${ }^{6}$, Jabbar Hussie Jr. ${ }^{7}, J^{2}$ nn Olinde ${ }^{8}$, \\ Aaron Shephard ${ }^{9}$, and Bradley Zelenka ${ }^{10}$ \\ ${ }^{1}$ University of Wisconsin-Madison \\ Madison, WI, USA \\ ${ }^{2}$ University of Minnesota-Twin Cities \\ Minneapolis, MN, USA \\ ${ }^{3}$ West Virginia University \\ Morgantown, $W V$, USA \\ ${ }^{4}$ University of Alaska-Fairbanks \\ Fairbanks, AK, USA \\ ${ }^{5}$ University of Mississippi \\ Oxford, MS, USA \\ ${ }^{6}$ University of South Carolina \\ Columbia, SC, USA \\ ${ }^{7}$ Delaware State University \\ Dover, DE, USA \\ ${ }^{8}$ Georgia Institute of Technology \\ Atlanta, GA, USA \\ ${ }^{9}$ Clemson University \\ Clemson, SC, USA \\ ${ }^{10}$ San Diego State University \\ San Diego, CA, USA
}

\begin{abstract}
The 2018 NASA Academy at Langley Research Center created proof-of-concept mixed reality (MR) and traditional simulators to enable quick vehicle concept prototyping and data collection for human factors studies. Urban Air Mobility (UAM) vehicle concepts were implemented as the focus for these simulators due to recent and dramatic rise in subject interest. The team used X-Plane 11 to model flight dynamics, and Unity for the virtual reality (VR) aspect of the MR simulator and to model the control panel in the traditional simulator. A physical version of the virtual control panel was also fabricated to provide haptic feedback to maintain the immersion for the MR simulator. ${ }^{1}$
\end{abstract}

\section{Introduction}

Since at least the 1940s, the concept of commercial UAM has existed in the United States. From 1949 to 1979, New York Airways transported people in helicopters between the major airports in New York City and various heliports throughout Manhattan [1]. Between 1941 and 1971, Los Angeles Airways offered similar services to numerous area locations including major airports and Disneyland [2]. Both urban transport services experienced multiple mechanical failures that injured or killed several dozens of passengers, crew members, and bystanders, forcing the programs to shut down $[3,4]$.

1. This research was made possible due to the funding of many Space Grant Consortia, including the Wisconsin Space Grant Consortium. 
Currently, leaders in industry and government are considering on-demand aviation as a solution to increasing urban ground traffic. In 2016, Uber Elevate published a feasibility study for an air taxi service. This paper describes operational challenges including ideal aircraft requirements (electric, low noise, capable of vertical take off and landing (VTOL)) and the necessary ground infrastructure (air traffic control requirements, vertiports) for a realizable service. In addition, potential benefits of urban air transportation are discussed. According to the paper, travel time from San Francisco's Marina to San Jose (normally a 2-hour commute by car) would be reduced to approximately 15 minutes using a VTOL air taxi [5]. In 2018, Uber Technology Inc. signed a second space act agreement with NASA. This partnership will focus specifically on computer modeling and simulating the impacts of small aircraft in crowded environments.

Recent advances in VR technology make VR and MR ideal tools for UAM simulation. Most realistic flight simulators are relatively expensive and bulky to use [6]. VR can accurately mimic flight conditions such as the controls and vistas, providing a fully immersive experience for the user at a reduced cost with minimal special requirements. MR, sometimes called hybrid reality, is the merging of virtual and real worlds to create new visualizations and settings [7]. Users in MR environments can interact with both physical and digital objects in real time. For example, a physical button can be placed in the same location as the corresponding button in the virtual environment. This provides haptic feedback and a more realistic experience as the user reaches to press the button in simulation. Due to the high stakes nature of aircraft production and flight training, VR and MR technologies can be powerful tools for the development of on-demand aviation.

\section{Flight Model}

\subsection{Design direction}

The aircrafts designed and modeled in this project are proof-of-concept for the rapid prototyping capabilities of the simulator. The design requirements of the flight vehicles were limited to a VTOL configuration, a payload of 1-4 passengers, and an airspeed of 135-200 mph during cruise, as specified by the Uber Elevate white paper [5]. The scope of the design includes basic sizing and power and the ability to fly and transition from vertical flight into horizontal flight. The scope excludes design considerations such as structures, precise aerodynamic analysis, transition analysis, and safety and redundancy features. Figure 1 outlines the design process taken to create the flight vehicles. First, the vehicle concepts were designed in NASA's OpenVSP to conduct basic vortex lattice method (VLM) aerodynamic analysis and to determine if the designs are aerodynamically efficient. From there, the designs are recreated in the commercial flight simulator X-Plane 11 Pro to model the flight dynamics. Finally, the flight data are fed in real-time from X-Plane to a visual model developed in the Unity game engine. 


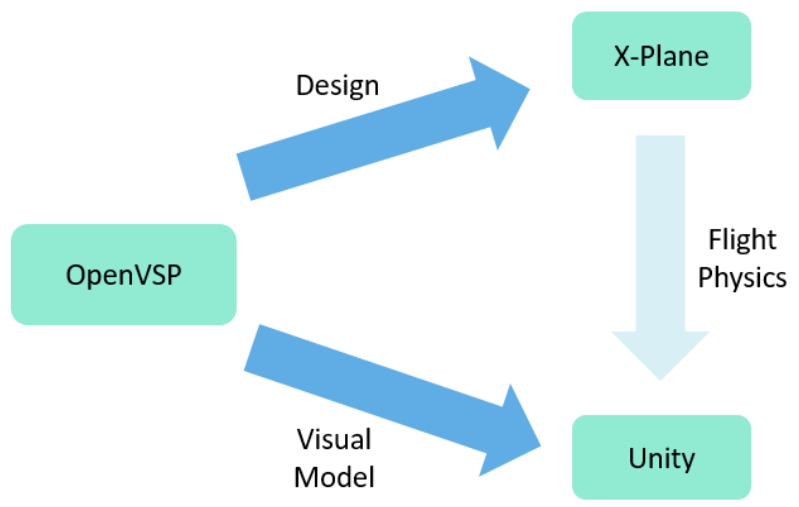

Fig. 1 Design process to model flight vehicles. Once the model is built in OpenVSP, it is traced into X-Plane Plane Maker. The 3D model from OpenVSP is also exported to Unity to be the visual model of the vehicle in the simulation.

The vehicle designs were created to fit the needs of UAM operations. Such aircraft need to be able to take off and land vertically to minimize ground infrastructure in highly populated areas. However, unlike a helicopter, these vehicles spend most of flight time traveling horizontally rather than hovering, and so the ability to transition to a fixed-wing configuration is essential to maximize efficiency. The use of redundant electric engines is also an improvement that compared to the noise a helicopter's combustion engine produces. The chosen configurations for the VTOL aircraft were a tilt-wing, tilt-rotor, and two hybrid designs between lift-plus-cruise and the other two configurations. These final two designs are commercial designs created in OpenVSP and X-Plane visually with pictures available online $[9,10]$. The team used commercial designs in order to demonstrate the quick prototyping capabilities of this simulator. Once OpenVSP and X-Plane Plane Maker were mastered, a design could be traced and implemented in under three hours. These designs are shown in Figure 2.
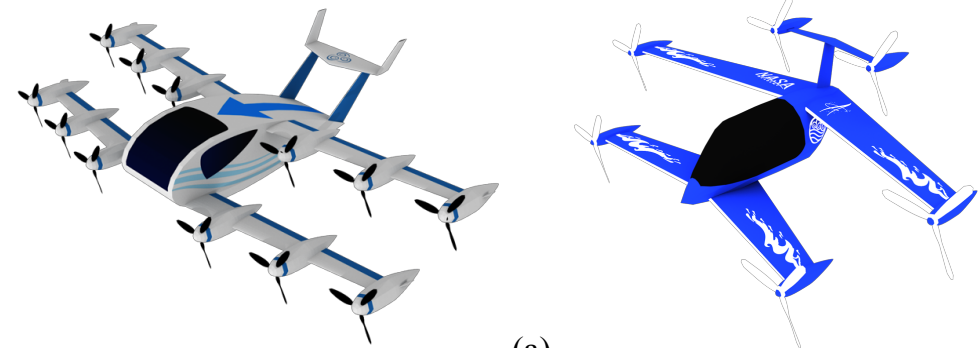

(a)

(b)
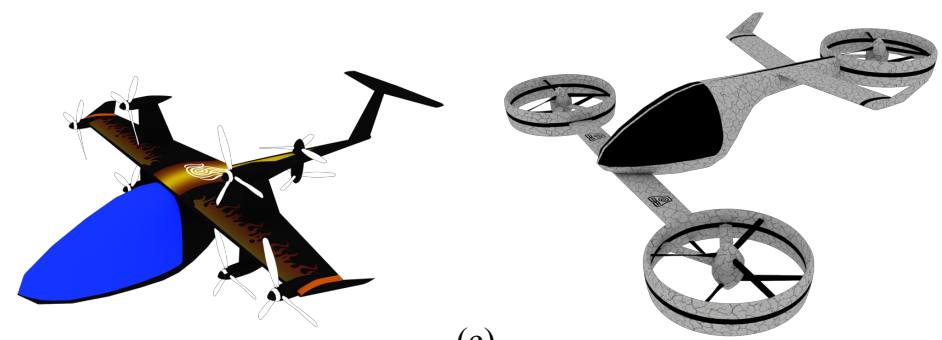

(c)

(d) 
Fig. 2 All four aircraft available to fly in simulator. (a) The AirfoilPlane, an original design the team created with the intention of having an airfoil-shaped fuselage; (b) The TM-1 Tilt-Rotor, an original design by the team - note the forward-swept wing, allowable because of the lack of aeroelastic analysis; (c) The AirSpaceX MOBi, and (d) The

Autonomous Flight Y6S, the two commercial designs the team chose to demonstrate the quick-prototyping capabilities of the simulator.

The team also needed to model a glass instrument and navigation panel that would provide the pilot with as real time data as possible. This was accomplished by taking the primary flight display and multi-function display from the Garmin-1000 that was already generated in X-Plane 11. The primary flight display provided altitude, attitude, and airspeed for the pilot. This display also controlled the autopilot that was configured for each aircraft. This display did have to be modified, as it showed the full range of airspace details and navigational aids used by general aviation pilots that are superfluous to more constrained UAM applications. These extra details were removed, leaving only the vertiports generated in World Editor and Unity superimposed upon some basic geographic imagery. The modified displays for the glass panels are shown in Figure 3.

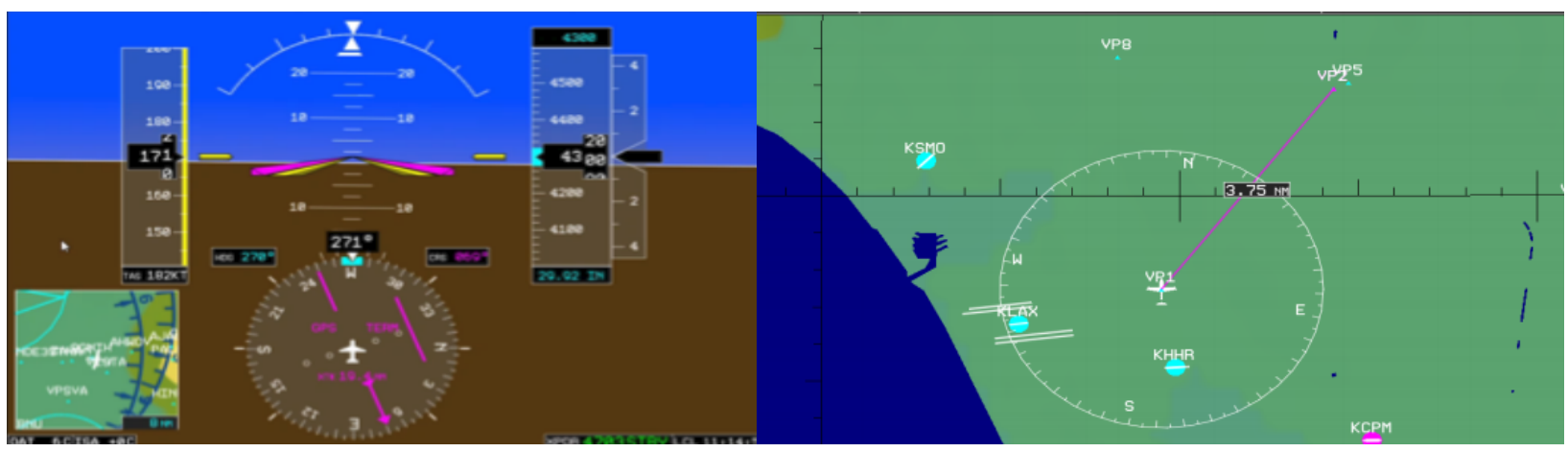

Fig. 3 (a) Primary Flight Display with Autopilot. (b) Multi-Function Display used for Navigation

\subsection{Vehicle design}

Initial sizing and weight for each vehicle was done by looking over commercial concepts available at the Vertical Flight Society's website, Electric VTOL News [8], and averaging available concepts' payload ratios, yielding a result of $25 \%$ payload ratio for each craft. Deviation from this value was limited, suggesting it is a reasonable enough approximation for the inertia of each vehicle. Similarly, comparing listed passenger and cargo capacities showed that most companies were allocating an average of $225 \mathrm{lbs}$ per passenger. Therefore, we could estimate the gross weight of each craft by assigning the number of passengers it should be capable of carrying. For example, a four passenger capacity amounts to a $900 \mathrm{lbs}$ payload, which translates to a craft weighing $3600 \mathrm{lbs}$ when fully loaded.

With the aircraft weight known, specific configuration design could begin. Once again using the Uber Elevate paper as a guide, the team sought to achieve 10 to $20 \mathrm{lb} / \mathrm{ft}^{2}$ wing and disk loading when sizing the wings and propellers [5]. The propulsion for each craft was sized using a MATLAB script the team developed, which took many configuration parameters obtained through VLM analyses in OpenVSP and output required power characteristics and propeller speeds. Electric motors are usually extremely efficient in a small range of speeds, and so each propulsion 
system was designed with the goal of having each propeller spin at the same speed whether in cruise or hover configuration. This was done by shutting off some propellers during horizontal flight. Additionally, since a primary goal of UAM vehicles is reduced noise, the propellers for each aircraft were sized such that the tip speed did not exceed Mach 0.8 in hover.

\subsection{Implementation}

Each vehicle design in X-Plane 11 was created using X-Plane Plane Maker. This program allowed the aeronautics team to recreate the key aircraft components (such as fuselage, wings, propulsion, etc.) from OpenVSP into a file that X-Plane could use to model the aircraft. Where necessary, the simulated aircraft deviated from the proposed UAM concept design guidelines. For example, the Uber Elevate white paper suggested that the vehicles use an electric propulsion system. This suggestion assumes advances in battery technology that are not yet actualized, which X-Plane Maker cannot model as it places a very conservative cap on the electric power available to vehicles. To circumvent this, turboprop engines were used in the aircraft designs to create the required power. Although these two types of engines do not perform exactly identically, only the overarching vehicle dynamics need to be realistically simulated at this stage, so this substitution was deemed acceptable.

\section{Virtual Environment Design}

\subsection{Virtual city environment design process}

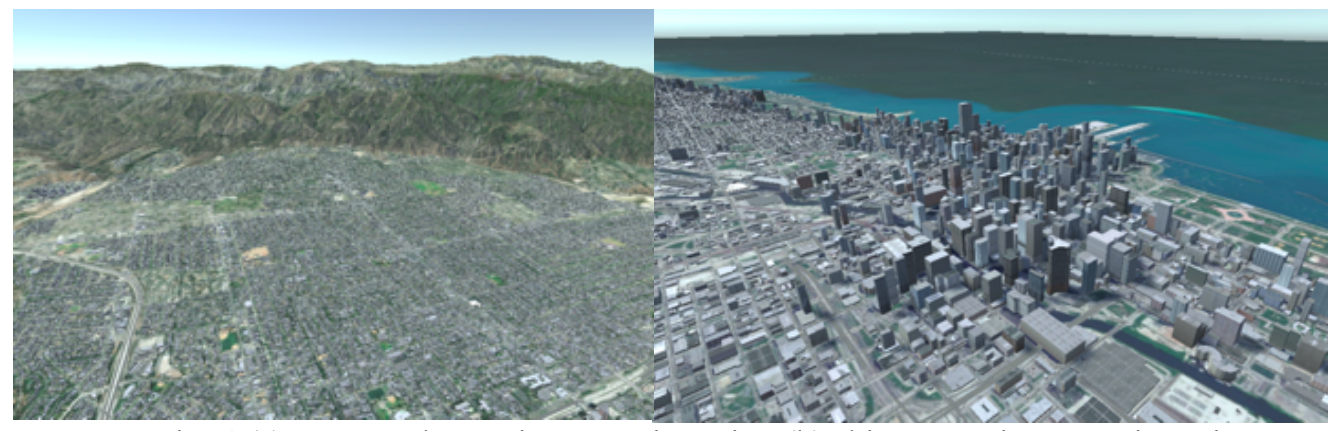

Fig. 4 (a)Los Angeles environment in Unity. (b)Chicago environment in Unity

A major part of the virtual environment design involved constructing city environments to aid UAM flight visuals. For this project, two real-world cities were replicated. Esri CityEngine, an advanced 3D city design software, was used to extract data from OpenStreetMap, a public volunteered geographic database, and generate city units modeled after the Google Map regions desired. The city units were then exported as .fbx files, imported into Unity, and then pieced together as a collective environment. Simple vertiports were created and then set on selected buildings. 12 vertiports were placed in each city providing 144 different flight paths for pilots. 


\section{Simulator Development}

\subsection{Physical simulator setup}

HTC Vive headsets were used to project the VR program to flight user. A LeapMotion hand tracker was connected and attached to the headset to accurately display the user's hand position while in the virtual environment. Figure 5 shows the DreamFlyer flight motion chair used to create pitch and roll to more accurately simulate pilot flight.

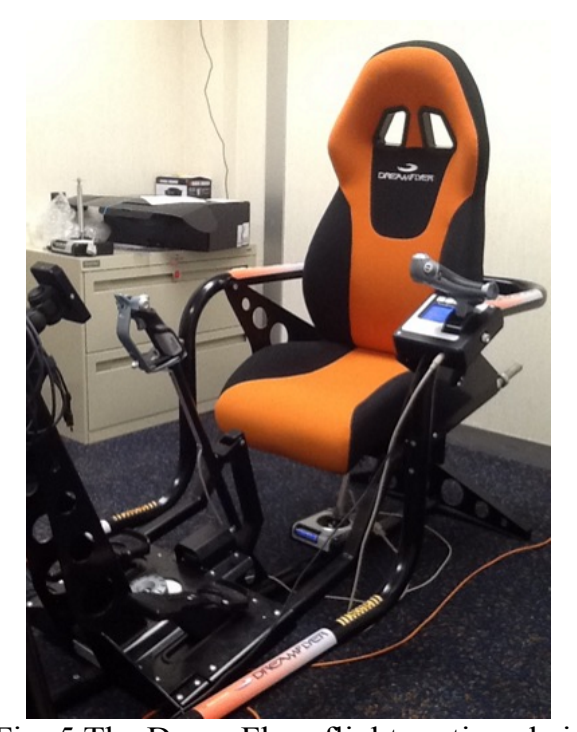

Fig. 5 The DreamFlyer flight motion chair

\subsection{Control board design process}

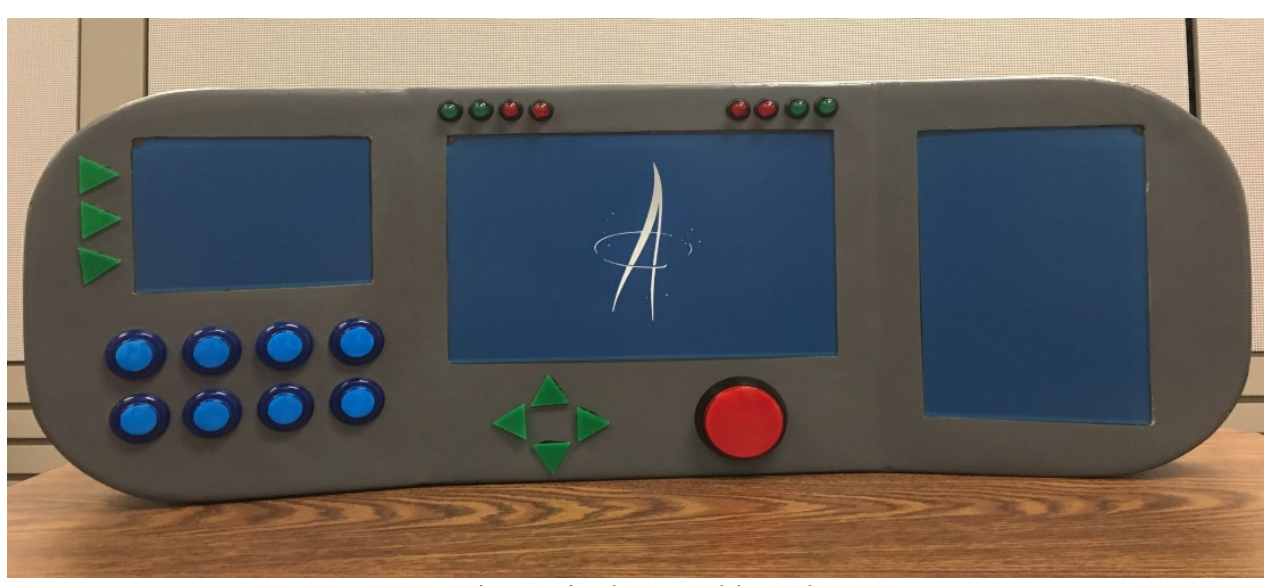

Fig. 6 Final control board

The control panel design was based on the idea that in the future VTOL aircraft will be as ubiquitous as cars, and therefore should be just as simple to pilot. The initial design was based on modern car dashboards and controls, expanded for use in simplified VTOL flight.

The initial outline was produced to scale in AutoCAD. From there, a cardboard prototype was put together with 3D printed buttons. The next step was to get pilot input on the control board 
design while it was attached to the DreamFlyer motion simulator. The control panel was then redesigned to further fit user needs and aircraft control requirements based on the input from the pilot, team members, and advisors. The rapid prototyping process required several iterations all including outlines produced in AutoCAD, 3D models generated in Fusion 360, and final models assembled with fiberboard faces. The materials used for the 3D model ranged from cardboard to poster board material to fiberboard. The back mounting was produced out of MakeBlock and a recycled monitor mount. Each prototype was mounted onto the DreamFlyer to test its spacing and functionality. Several prototypes were designed, assembled, and tested in the process of developing the final design. The final design was produced in the Langley modeling shop with high density foam covered in fiberglass and sealed with resin. Painting was done by the modeling shop, and other installation was done by the mechanical team and an electrical lead. The final model seen in Figure 6 included buttons with functional electronics as well as artificial screens, in order to perpetuate immersion for the user in the MR environment.

\subsection{Control board software}

Figure 7 outlines the software process developed to translate physical control board actions into digital simulator actions. Each panel button, when pressed, generated a unique identifier code via the Arduino microcontroller. The code was passed through the serial port to Unity's software environment. Unity then matched the identifier to pre-mapped X-Plane keyboard commands based on the aircraft design selected by the user. The appropriate keyboard presses were then sent to XPlane using the Windows Input Simulator function library. The simulated key presses caused actions in X-Plane's program which lead the same action to occur in the UAM virtual environment.

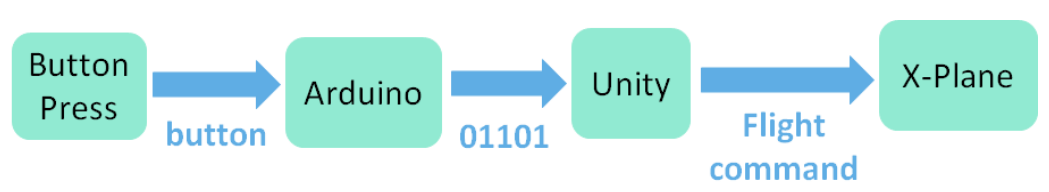

Fig. 7 Software process outline for control panel button presses

\subsection{Simulator software}

The simulator software is run through an executable file built using the Unity Engine and plugins to make that software take advantage of the VR hardware in an efficient manner. As described in Section 3, the program X-Plane supplies flight physics for the UAM simulator. It does so using a NASA developed plugin called X-Plane Connect. In addition, the graphics for the control panel monitors and displays are also pulled from X-Plane. The executable file produced takes in command line variables that allow a user to toggle the customization options described above, such as weather, location, and vehicle choice. These command line variables are taken advantage of when launching from the graphical user interface (GUI).

\section{X-Plane Connect}

Plugins can be developed for the X-Plane program, allowing streamlined access and manipulation of data variables. These variables include aircraft $\mathrm{X}, \mathrm{Y}$, and $\mathrm{Z}$ coordinates, pitch, roll, yaw, vector thrust angle, throttle, and indicated airspeed. X-Plane Connect enabled a running instance of the X-Plane program to send its data variables to an aircraft model in the Unity based 
UAM simulator. A dynamic link library (DLL) file was written to help translate the $\mathrm{C} / \mathrm{C}++$ code of X-Plane Connect to the $\mathrm{C} \#$ code of the Unity game engine.

Using the DLL, X-Plane, and X-Plane Connect, an aircraft's location and flight dynamics could be determined in the UAM simulator. This was done by converting changes in the X-Plane data variables into motion commands for the simulator aircraft. The motion of the VR aircraft mirrored the motion of the X-plane model.

\section{Results}

The designed cities and aircraft were successfully modeled and transferred into the Unity game engine. Combining these models with the flight motion chair, hand tracker, and physical control panel created an immersive flight simulator for UAM environments. Users could choose between two locations, four aircraft, and three types of weather creating 24 different scenarios to fly in. Utilizing X-Plane Connect to transfer flight data from X-Plane to the UAM simulator allowed users to switch between VTOL and horizontal flight modes with relatively realistic response times and flight dynamics. Matching the positions of the virtual throttle, center stick, and cockpit panel to their physical counterparts gave users sufficient haptic feedback while controlling the aircraft. Additionally, users could feel some proprioceptive feedback to the virtual environment due to changes in their physical pitch and roll caused by the DreamFlyer.

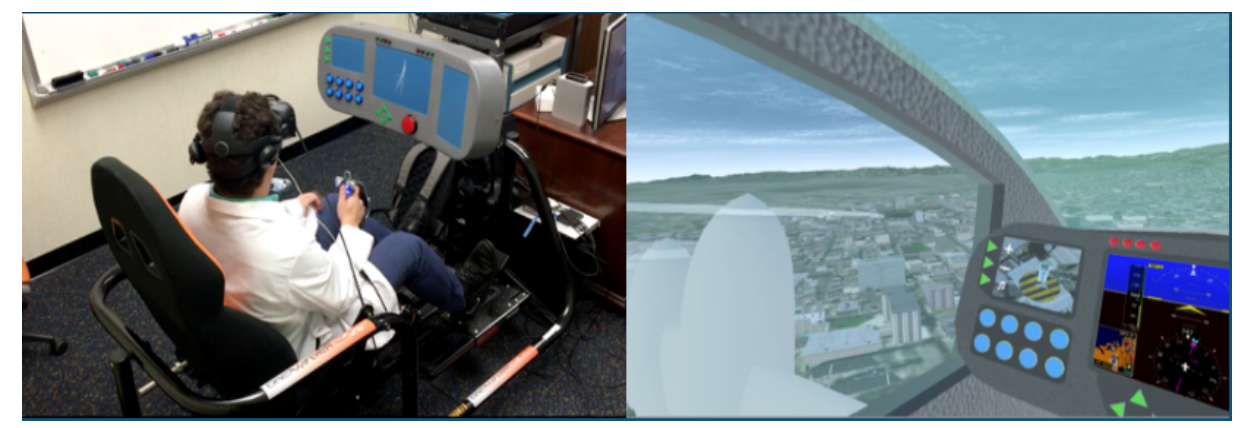

Fig. 8 The virtual and mixed reality environments of the UAM simulator

As mentioned in section 5.3, the UAM simulator is tied directly to a running instance of X-Plane. Tests showed that an inadvertent event in X-Plane, such as a crash, would disrupt the UAM simulator as well. Changes in the user's physical pitch and roll are purely dependent on the DreamFlyer's center stick position. However, changes in the pilot's virtual attitude are determined by control inputs and aerodynamic factors. Occasionally this caused a mismatch between the actual and perceived pilot attitude. Users would sometimes feel changes in their position before seeing the changes in the VR environment. Although different weather patterns could be implemented in the current UAM simulator, they ultimately did not affect how aircraft flew in the virtual environment. This can be improved upon in future iterations of the simulator.

\section{Conclusions/Future Work}

In ten weeks, the 2018 NASA Academy at Langley completed the necessary groundwork for creating a realistic simulator of Urban Air Mobility concepts. Initial trials show that the combination of virtual and mixed reality elements create a somewhat accurate replica of what 
UAM pilots will experience during flight. Some environmental improvements to consider in future simulator versions are the addition of more cities for users to fly in and weather patterns that directly influence aircraft flight dynamics. Also, optimizing the simulator software to reduce central processing unit (CPU) and graphics processing unit (GPU) usage will greatly improve user experience.

Ideally, this project will have future applications in UAM pilot behavior research. Integrating biometric sensors to the current simulator could help determine a pilot's physical and emotional states throughout various flight scenarios. A simple example is the addition of an eye tracker to the VR headset. This would provide valuable data on the pilot's gaze pattern during both normal UAM flights and simulated emergency events such as an engine failure or catastrophic weather.

\section{Acknowledgement}

We would like to thank our Technical Advisory Committee at Langley Research Center: Mark Agate, Ken Goodrich, Nelson Guerreiro, Sam James, Kellie Kennedy, Jon Levy, Neil O’Conner, Joey Ponthieux, Garry Qualls, Patrick Quach, Kyle Smalling, Lawrence Taylor, Elizabeth Ward.

This research was supported by the National Aeronautics and Space Administration along with multiple state Space Grant Consortiums.

\section{References}

[3] "Aircraft Accident Report - New York Airways, inc., Boeing-Vertol 107-II, N6673D, New York International Airport, Jamaica, New York October 14, 1963", National Transportation and Safety Board, June 1964.

[9] "AirSpaceX MOBi," Electric VTOL News by the Vertical Flight Society, URL: http://evtol.news/aircraft/airspacex/ [retrieved 10/9/2018].

[10]“Autonomous Flight Y6S," Electric VTOL News by the Vertical Flight Society, URL: http://evtol.news/aircraft/autonomous-flight/ [retrieved 10/9/2018].

[7] de Souza e Silva, Adriana; Sutko, Daniel M.. Digital Cityscapes: merging digital and urban playspaces, Peter Lang Publishing Inc., New York, 2009

[8]"Electric VTOL News," The Vertical Flight Society, URL: http://evtol.news/ [retrieved 10/9/2018].

[5] Holden, J., and Goel, N., "Fast-Forwarding to a Future of On-Demand Urban Air Transportation," Uber Elevate [White Paper], URL: https://www.uber.com/elevate.pdf [retrieved 25 July 2018]. 
[6] "How Reality Technology is Used in Aviation", Reality Technologies, URL: http://www.realitytechnologies.com/aviation [retrieved 10 June 2018]

[2] Staff, S.B. "Terminal For Copter Air Express in U.S.", San Bernardino Daily Sun, Vol. 60, No. 94, 1957, p 13.

[4] Thipphavong, D. P., Apaza, R., Barmore, B., Battiste, V., Burian, B., Dao, Q., Feary, M., Go, S., Goodrich, K. H., Homola, J., Idris, H. R., Kopardekar, P. H., Lachter, J. B., Neogi, N. H., Ng, H. K., Oseguera-Lohr, R. M., Patterson, M. D., and Verma, S. A., "Urban Air Mobility Airspace Integration Concepts and Considerations", 2018 Aviation Technology, Integration, and Operations Conference, AIAA AVIATION Forum, (AIAA 2018-3676).

[1] "World Airline Directory." Flight International. May 3, 1957. 608. 